\title{
A Model Predictive Control Scheme with Additional Performance Index for Transient Behavior
}

\author{
Andrea Alessandretti ${ }^{1}{ }^{2}$, António Pedro Aguiar ${ }^{3}$ and Colin N. Jones ${ }^{1}$
}

\begin{abstract}
This paper presents a Model Predictive Control (MPC) scheme for nonlinear continuous time systems where an extra performance index, which is not a measure of the distance to the set point, is introduced to influence the transient behavior of the controlled system. The scheme is based on the following fact, proven in the paper: Given a stabilizing MPC controller, adding a function, integrable in the interval $[t,+\infty)$, to the stage cost does not change the asymptotic convergence property of the closed loop state trajectory. As a numerical example, this result is applied to solve a simple visual servo control problem where an MPC controller drives the state to the origin while penalizing weakly observable trajectories.
\end{abstract}

\section{INTRODUCTION}

This paper addresses the design of a state feedback sample-data MPC controller for continuous time nonlinear systems where an additional performance index is introduced to modify the transient behavior.

In classic MPC schemes, at every sampling time, the controller selects among all the possible feasible future input trajectories of the system, the control signal that minimizes a given performance index. Then, according to the receding horizon formulation, the initial part of such input signal is applied to the plant and, once the next sampling time is reached, the process is repeated. Many MPC schemes have been proposed in the literature, we refer to [15], [14] and, more recently, [17], [10] for an overview. From the class of quasi-infinite horizon schemes, where both terminal cost and a terminal set are employed, we refer to [3], [7] and [12] (where the terminal constraint is implicitly satisfied). In [16], [9], [11] the terminal set is dropped. In that case, for these unconstrained MPC schemes, the emphasis is on the computation of a "sufficiently long" horizon length that ensures stability. In [18] the authors propose an interesting unifying scheme that borrows from both previous families.

In the papers mentioned above, and in the majority of MPC schemes, the performance index is chosen to be a measure of the distance form the desired steady state. Therefore, selecting the input trajectory that minimizes such distance, together with an appropriate selection of the MPC design parameters (e.g., terminal set, terminal cost, horizon

*This work was supported by projects CONAV/FCT-PT [PTDC/EEACRO/113820/2009], MORPH [EU FP7 ICT 288704], and FCT [PEst-OE/EEI/LA0009/2011]. The first author benefited from grant SFRH/BD/51073/2010 of the Foundation for Science and Technology (FCT), Portugal.

${ }^{1}$ École Polytechnique Fédérale de Lausanne (EPFL), Lausanne, Switzerland.

2 Institute for Systems and Robotics (ISR), Instituto Superior Tecnico (IST), Lisbon, Portugal.

3 Faculty of Engineering, University of Porto (FEUP), Porto, Portugal. length), the MPC controller drives the state to the desired equilibrium point. There are however many applications where it is desirable to have a more flexible performance index that does not take into account only the input energy and the distance to the desired steady-state trajectory. As an example, in [2] the authors address the problem of stabilizing a system avoiding weakly observable closed loop trajectories and propose, among other solutions, an MPC controller where an observability index that penalizes weakly observable trajectories is introduced in the stage cost.

Prompted by this observation, in this work we propose an MPC scheme where an additive stage cost, which determines the desired transient behavior of the system, is introduced. As a main result, we show that, if the additive function is integrable in the interval $[t,+\infty)$, where $t$ is the current time, the convergence of the closed loop state trajectory to the set point is not compromised.

The remaining of this paper is organized as follows: The MPC law and the control problem is introduced in Section II. Section III contains the main result, followed by Section IV where some considerations on the design of the additive stage cost and on stability and convergence of the proposed method are discussed. An application of this result is presented in Section V, where similarly to [2], an MPC controller is used to drive the state of a system to the origin while penalizing weakly observable trajectories. Although, in contrast to [2], the observability index is used to influence only the transient behavior and, thus, convergence to the origin can be established.

\section{PROBLEM DEFINITION}

Consider the dynamic system

$$
\dot{x}(t)=f(t, x(t), u(t)), \quad x(0)=x_{0}, \quad t \geq t_{0}
$$

where $x(t) \in \mathbb{R}^{n}$ and $u(t) \in \mathbb{R}^{m}$ are the state and the input vectors at time $t$, respectively, and $x_{0}$ and $t_{0}$ are the initial state and time, respectively.

The system is subject to the following constraints

$$
x(t) \in \mathcal{X}(t) \subseteq \mathbb{R}^{n}, \quad u(t) \in \mathcal{U}(t) \subseteq \mathbb{R}^{m}, \quad t \geq 0,
$$

where the set-valued maps $\mathcal{X}: \mathbb{R} \rightrightarrows \mathbb{R}^{n}$ and $\mathcal{U}: \mathbb{R} \rightrightarrows \mathbb{R}^{m}$ denote the time varying state and input constraints set, respectively. Next, we define the MPC optimization problem $\mathcal{P}(t, z)$, with $(t, z) \in \mathbb{R} \times \mathcal{X}(t)$, where for a generic trajectory $x(\cdot)$, we denote by $x\left(\left[t_{1}, t_{2}\right]\right)$ the trajectory considered in the time interval $\left[t_{1}, t_{2}\right]$ and we use the notation $x(\cdot ; t, z)$ whenever we want to make explicit the dependence of the trajectory $x(\cdot)$ on the optimization 
problem parameters $t$ and $z$. For sake of simplicity, the dependence on time and parameters is dropped whenever clear from the context.

Definition 1: (MPC problem) Given a pair $(t, z) \in \mathbb{R} \times \mathbb{R}^{n}$ and a horizon length $T \in \mathbb{R}_{>0}$, the open loop MPC optimization problem $\mathcal{P}(t, z)$ consists of finding the optimal control signal $\bar{u}^{*}([t, t+T])$ that solves

$$
\begin{aligned}
& J_{T}^{*}(t, z)=\min _{\bar{u}([t, t+T])} J_{T}(t, z, \bar{u}([t, t+T])) \\
& \text { s.t. } \quad \dot{\bar{x}}(\tau)=f(\tau, \bar{x}(\tau), \bar{u}(\tau)) \quad \forall \tau \in[t, t+T] \\
& \bar{x}(t)=z \\
& \bar{x}(\tau) \in \mathcal{X}(\tau) \\
& \bar{u}(\tau) \in \mathcal{U}(\tau) \\
& \bar{x}(t+T) \in \mathcal{X}_{f}(t+T)
\end{aligned}
$$

with

$$
\begin{aligned}
& J_{T}(t, x, \bar{u}([t, t+T]))= \\
& \int_{t}^{t+T} l(\tau, \bar{x}(\tau), \bar{u}(\tau)) d \tau+F(T, \bar{x}(T)) .
\end{aligned}
$$

The finite horizon cost $J_{T}(\cdot)$ is composed of the stage cost $l: \mathbb{R} \times \mathbb{R}^{n} \times \mathbb{R}^{m} \rightarrow \mathbb{R}_{\geq 0}$ and the terminal cost $F: \mathbb{R} \times \mathbb{R}^{n} \rightarrow \mathbb{R}_{\geq 0}$, which is defined over the time varying terminal set $\overline{\mathcal{X}}_{f}: \mathbb{R} \rightrightarrows \mathbb{R}^{n}$. We denote by $k_{f}: \mathbb{R} \times \mathbb{R}^{n} \rightarrow \mathbb{R}^{m}$ a feasible auxiliary control law defined over the terminal set, i.e., $\quad k_{f}(t, x(t)) \in \mathcal{U}(t)$ with $x(t) \in \mathcal{X}_{f}(t), \forall t \geq t_{0}$. In a sample-data receding horizon strategy, the control input is computed at discrete sample times $\mathcal{T}:=\left\{t_{0}, t_{1}, \ldots\right\}$, and the MPC control law is defined as

$$
u(t)=k_{M P C}(t, x):=\bar{u}^{*}(t ;\lfloor t\rfloor, x(\lfloor t\rfloor)),
$$

where $\lfloor t\rfloor$ is the maximum sampling time $t_{i} \in \mathcal{T}$ smaller or equal than $t$, i.e., $\lfloor t\rfloor=\max _{i}\left\{t_{i} \in \mathcal{T}: t_{i} \leq t\right\}$. This work addresses the problem of designing an MPC controller that (i) asymptotically drives the state to the origin and (ii) accepts, as design input, a performance index for the transient behavior. Toward this goal, the stage cost is defined as

$$
l(t, x, u):=l_{1}(t, x, u)+l_{2}(t, x, u) .
$$

where the primary stage cost $l_{1}: \mathbb{R} \times \mathbb{R}^{n} \times \mathbb{R}^{m} \rightarrow \mathbb{R}_{\geq 0}$ defines the desired asymptotic behavior of the closed loop system, as will be more clear later, and the secondary stage cost $l_{2}: \mathbb{R} \times \mathbb{R}^{n} \times \mathbb{R}^{m} \rightarrow \mathbb{R}_{\geq 0}$ characterizes the desired transient behavior.

\section{MAIN RESULT}

The main assumption of the proposed method is the following:

Assumption 1: The secondary stage cost function $l_{2}(\cdot)$ is uniformly upper bounded by a function $b: \mathbb{R} \rightarrow \mathbb{R}$, with $b(\cdot)$ integrable in the interval $\left[t_{0}, \infty\right)$, i.e., $l_{2}(t, x, u) \leq b(t), \quad \forall t \geq t_{0}, \quad x \in \mathcal{X}(t), u \in \mathcal{U}(t)$ and

$$
\mathcal{L}(t):=\int_{t}^{\infty} b(\tau) d \tau<+\infty, \quad \forall t \geq t_{0}
$$

The bound in (6) guarantees that $l_{2}(\cdot)$ can influence the behavior of the MPC controller in the transient phase, leaving the function $l_{1}(\cdot)$ to determine the asymptotic closed loop behavior. In Section IV-A we provide a meaningful class of functions that satisfy (6).

Before stating the main result we introduce some assumptions, which are common in the MPC literature.

Assumption 2: The function $f(\cdot)$, introduced in (1), is locally Lipschitz continuous in $x$ and piecewise continuous in $t$ and $u$ in the region of interest. Moreover, without loss of generality, it satisfies $f(\cdot, 0,0)=0$.

Assumption 2, guarantee the existence and, together with the boundedness of the state trajectory $x(\cdot)$ resulting from Theorem 1, uniqueness of the solution $x(\cdot)$ of the initial value problem (1) in closed-loop with the proposed controller. Note that if the system has an equilibrium point different from the origin, it is always possible to perform a change of coordinates such that the origin of the system expressed in the new coordinates is an equilibrium point. In general, the region of interest coincides with the desired region of attraction.

Assumption 3 (Initial feasibility): The optimization problem $\mathcal{P}\left(t_{0}, x_{0}\right)$ admits a feasible solution.

Assumption 4 (Sufficient conditions for convergence):

(i) The state constraint set $\mathcal{X}(\cdot)$ and the terminal set $\mathcal{X}_{f}(\cdot) \subseteq \mathcal{X}(\cdot)$ are closed, connected, and contain the origin. Moreover, the input constraints set $\mathcal{U}(\cdot)$ is compact with $0 \in \mathcal{U}(\cdot)$.

(ii) The primary stage cost satisfies $l_{1}(\cdot, 0,0)=0$ and there is a continuous positive definite and radially unbounded $^{1}$ function $M: \mathbb{R}^{n} \rightarrow \mathbb{R}_{\geq 0}$ such that $l_{1}(t, x, u) \geq M(x)$ for all $(t, x, u) \in \mathbb{R} \times \mathbb{R}^{n} \times \mathbb{R}^{m}$.

(iii) The function $F(\cdot)$ is positive semi-definite and continuously differentiable almost everywhere ${ }^{2}$.

(iv) There exists a feasible control law $k_{f}: \mathbb{R} \times \mathbb{R}^{n} \rightarrow \mathbb{R}^{m}$, defined over the terminal set $\mathcal{X}_{f}(\cdot) \subseteq \mathbb{R}^{n}$, such that, for the closed loop system (1) with $u(t)=k_{f}(t, x)$, the state and input vectors are such that $x(t) \in \mathcal{X}_{f}(t) \subseteq \mathcal{X}(t)$ and $u(t) \in \mathcal{U}(t)$, respectively, and the following cost decrease condition holds:

$$
\begin{aligned}
\dot{F}(t, x) & =F_{t}(t, x)+F_{x}(t, x) f\left(t, x, k_{f}(t, x)\right) \\
& \leq-l_{1}\left(t, x, k_{f}(t, x)\right)
\end{aligned}
$$

for all $t \geq t_{0}$ and $x$ with $F(\cdot)$ differentiable at $(t, x)$ and initial conditions $\left(t_{0}, x_{0}\right) \in \mathbb{R} \times \mathbb{R}^{n}$, with $x_{0} \in \mathcal{X}_{f}\left(t_{0}\right)$.

${ }^{1}$ A function $f: \mathbb{R}^{n} \rightarrow \mathbb{R}$ is said to be radially unbounded if $f(x) \rightarrow \infty$ as $\|x\| \rightarrow \infty$.

${ }^{2}$ A property said to hold "almost everywhere" if the set of elements for which the property does not hold is a set of Lebesgue measure zero. 
The terms $F_{t}(t, x)$ and $F_{x}(t, x)$ denote the partial derivatives of $F(\cdot)$ with respect of the time and the state variables, respectively. Considering condition (7) to hold almost everywhere allows the use a terminal cost that is non differentiable in some points (e.g., $F(x)=\|x\|$ ), which can be useful in the design phase. It is important to stress that replacing $l_{1}(\cdot)$ with $l(\cdot)$ in Assumption 4 we obtain the well known sufficient conditions for convergence to the origin of the MPC strategy (see, e.g., [6], [7], [4], [1] for similar results). It turns out, as stated in the following Theorem, that Assumptions 1-4 are sufficient to prove convergence for the proposed strategy, i.e., the convergence to the origin is not compromised by adding an extra cost with bounded integral over $[t, \infty)$ to the stage cost of a stable MPC controller.

Theorem 1: Consider system (1) in closed loop with (4), where $l(\cdot)$ is decomposed as (5), and suppose that Assumptions 1-4 hold. Then, the vector $x(t)$ converges to zero as $t \rightarrow \infty$ with region of attraction consisting of the set of states $x$ for which $\mathcal{P}\left(t_{0}, x\right)$, introduced in Definition 1, admits a feasible solution.

Proof: Consider the optimization problem $\mathcal{P}(\hat{t}, \hat{x})$ and assume that it admits a feasible solution. Using the minimizer $\bar{u}^{*}(\cdot ; \hat{t}, \hat{x})$, and the associated state trajectory $\bar{x}^{*}(\cdot ; \hat{t}, \hat{x})$, we define the following function

$$
\begin{aligned}
& V(\hat{t}, \hat{x}):=\int_{\hat{t}}^{\hat{t}+T} l_{1}\left(\tau, \bar{x}^{*}, \bar{u}^{*}\right)+l_{2}\left(\tau, \bar{x}^{*}, \bar{u}^{*}\right) d \tau \\
& \quad+F\left(\hat{t}+T, \bar{x}^{*}(\hat{t}+T)\right)+\mathcal{L}(\hat{t}+T)
\end{aligned}
$$

where, in contrast to some classical other functions used to prove convergence (e.g., [3], [4], [8]), here we introduce the term $\mathcal{L}(\cdot)$. Let $u_{e_{i}}(t, x)$ be the extended input trajectory obtained as a concatenation of the optimal control input computed at time $t_{i}$ with the auxiliary law $k_{f}(\cdot)$, i.e., $u_{e_{i}}(t, x):=\left\{\begin{array}{ll}\bar{u}^{*}\left(t ; t_{i}, x_{i}\right) & t \in\left[t_{i}, t_{i}+T\right] \\ k_{f}(t, x) & t>t_{i}+T\end{array}\right.$, where $x_{i}=x\left(t_{i}\right)$, and let $x_{e_{i}}(\cdot)$ be the associated extended state trajectory, thus $x_{e_{i}}(t)=\bar{x}^{*}\left(t ; t_{i}, x_{i}\right)$, with $t \in\left[t_{i}, t_{i}+T\right]$. Note that, from Assumption 4 (iv), the trajectories $x_{e_{i}}(\cdot)$ and $u_{e_{i}}(\cdot)$ are always feasible, i.e., they satisfy (2). Next, we prove that, for any pair $\left(t_{i}, x_{i}\right)$ such that the optimization problem $\mathcal{P}\left(t_{i}, x_{i}\right)$ admits a feasible solution and for any $\delta \geq 0$, the following cost decrease inequality holds along the extended state trajectory: $V\left(t_{i}+\delta, x_{e_{i}}\left(t_{i}+\delta\right)\right) \leq V\left(t_{i}, x_{i}\right)-\int_{t_{i}}^{t_{i}+\delta} M\left(x_{e_{i}}(\tau)\right) d \tau$. To this end, let first consider $\delta \leq T$.

$$
\begin{aligned}
& V\left(t_{i}+\delta, x_{e_{i}}\left(t_{i}+\delta\right)\right) \leq \int_{t_{i}+\delta}^{t_{i}+T+\delta} l\left(\tau, x_{e_{i}}, u_{e_{i}}\right) d \tau \\
& \quad+F\left(t_{i}+T+\delta, x_{e_{i}}\left(t_{i}+T+\delta\right)\right)+\mathcal{L}\left(t_{i}+T+\delta\right) \\
& =V\left(t_{i}, x_{e_{i}}\left(t_{i}\right)\right)-\int_{t_{i}}^{t_{i}+\delta} l\left(\tau, \bar{x}^{*}, \bar{u}^{*}\right) d \tau \\
& \quad+\int_{t_{i}+T}^{t_{i}+T+\delta} l_{1}\left(\tau, x_{e_{i}}, u_{e_{i}}\right) d \tau+\int_{t_{i}+T}^{t_{i}+T+\delta} l_{2}\left(\tau, x_{e_{i}}, u_{e_{i}}\right) d \tau \\
& \quad+F\left(t_{i}+T+\delta, x_{e_{i}}\left(t_{i}+T+\delta\right)\right)-F\left(t_{i}+T, x_{e_{i}}\left(t_{i}+T\right)\right) \\
& \quad+\mathcal{L}\left(t_{i}+T+\delta\right)-\mathcal{L}\left(t_{i}+T\right)
\end{aligned}
$$

where the first inequality arises from the fact that (i) the extended trajectory is not optimal, and (ii) the minimizer of $J_{T}(\cdot)$ correspond with the minimizer of $V(\cdot)$ since, for a given time $t$, the two functions differ only by a constant term. Combining this with fact that $\int_{t_{i}+T}^{t_{i}+T+\delta} l_{2}(\cdot) d \tau \leq$ $\int_{t_{i}+T}^{t_{i}+T+\delta} b(\tau) d \tau=\mathcal{L}\left(t_{i}+T\right)-\mathcal{L}\left(t_{i}+T+\delta\right)$ and $\quad-\int_{t_{i}+T}^{t_{i}+T+\delta} l_{1}\left(\tau, x, k_{f}(\tau, x)\right) d \tau \quad \geq$ $F\left(t_{i}+T+\delta, x\left(t_{i}+T+\delta\right)\right)-F\left(t_{i}+T, x\left(t_{i}+T\right)\right)$, which is obtained integrating both sides (7) from $t_{i}+T$ to $t_{i}+T+\delta$, results in

$$
\begin{aligned}
& V\left(t_{i}+\delta, x_{e_{i}}\left(t_{i}+\delta\right)\right)-V\left(t_{i}, x_{e_{i}}\left(t_{i}\right)\right) \\
& \quad \leq-\int_{t_{i}}^{t_{i}+\delta} l\left(\tau, \bar{x}^{*}, \bar{u}^{*}\right) d \tau \leq-\int_{t_{i}}^{t_{i}+\delta} M\left(x_{e_{i}}\right) d \tau .
\end{aligned}
$$

where the last where the last inequality follows from Assumption 4 (ii) and the fact that $l_{2}(\cdot)$ is nonnegative. Using similar computations it is easy to conclude that the same result applies for the case where $\delta>T$.

Inequality (9) proves the decrease of the function $V(\cdot)$ along the extended trajectory. Next, we show that the same applies to the closed-loop (1) with (4), i.e., using recursively the MPC optimization problem in the receding horizon fashion. To this end, we first observe that the state trajectory $x(\cdot)$ of the closed-loop (1) with (4) can be viewed as a concatenation of pieces of extended trajectories, which means that

$$
\begin{aligned}
& V(t, x(t))-V\left(t_{0}, x_{0}\right) \\
& \leq-\sum_{j=0}^{i-1} \int_{t_{j}}^{t_{j+1}} M\left(x_{e_{j}}(\tau)\right) d \tau-\int_{t_{i}}^{t} M\left(x_{e_{i}}(\tau)\right) d \tau \\
& =-\int_{t_{0}}^{t} M(x(\tau)) d \tau
\end{aligned}
$$

with $t_{i}=\lfloor t\rfloor$. Note that the optimization problem $\mathcal{P}\left(t_{i}, x\left(t_{i}\right)\right)$ is recursively feasible since, by Assumption 3 , it is feasible at time $t_{0}$ and the extended input keeps it feasible for all $\delta>0$ and, specifically, for the generic interval from $t_{i}$ to $t_{i+1}$. From (10) we have that any level set of $V(t, x)$, which are always compact sets from $M(\cdot)$ being radially unbounded, is invariant. Thus the closed loop trajectory $x(\cdot)$ is bounded and confined within the time varying bounded set $\left\{x: V(t, x) \leq V\left(t_{0}, x_{0}\right)\right\}$ for $t \geq t_{0}$. At this point, in order to prove convergence to the origin, we use the Barbalat's lemma (e.g., Lemma 8.2 in [13]). Note that $M(x(t))$ is a uniformly continuous function of $t$ because it is continuous in $x$ with $x$ bounded and $x$ is uniformly continuous in $t$, since from Assumption 4 (i), $u$ is bounded. Moreover, from (10) we have that

$$
\lim _{\delta \rightarrow \infty} \int_{t_{0}}^{t_{0}+\delta} M(\tau) d \tau<+\infty
$$

where the limit exists since the function $\int_{t_{0}}^{t_{0}+\delta} M(\tau) d \tau$ is increasing in $\delta$ and, from (10), upper bounded. Thus, by Barbalat's lemma, $M(x(t)) \rightarrow 0$ as $t \rightarrow 0$ and, by the positive-definitiveness of $M(\cdot)$, the state vector $x(t) \rightarrow 0$ with $t \rightarrow 0$, which concludes the proof. 


\section{CONSIDERATIONS}

This section contains a discussion on the implications of the result presented in the previous section. In particular, we propose a design technique for the secondary stage cost $l_{2}(\cdot)$ that complies with Assumption 1. The convergence and Lyapunov stability of the proposed MPC scheme are also discussed.

\section{A. Design of the secondary stage cost}

Let $g(t, x, u)$ be a performance index for the transient behavior that we wish to minimize. If Assumption 1 with $l_{2}(\cdot)=g(\cdot)$ cannot be a priori verified, a simple procedure is to introduce a function $p(\cdot)$, with $\int_{t}^{\infty} p(\tau) d \tau<+\infty, \forall t \geq t_{0}$, and

$$
l_{2}(\cdot)=\operatorname{sat}_{S}(g(\cdot)) p(t), \quad \operatorname{sat}_{S}(x)= \begin{cases}0 & \text { if } x<0, \\ x & \text { if } 0 \leq x \leq S, \\ S & \text { if } x>S\end{cases}
$$

for some arbitrarily big $S \in[0,+\infty)$. Broadly speaking, $p(\cdot)$ modulates $g(\cdot)$ that is previously saturated and Assumption 1 is clearly satisfied. Note that, in general it is desirable to choose a big value for the constant $\mathrm{S}$ since, depending on on the solver used to solve the optimization problem $\mathcal{P}(\cdot)$, a saturated value of $g(\cdot)$ might be considered as a constant and, thus, have no impact on the optimal solution.

Considering that the goal of $l_{2}(\cdot)$ is to influence the transient behavior, a natural choice for the function $p(\cdot)$ is the an exponential function $p(t)=B e^{-\alpha t}$, where the design parameters $B \in \mathbb{R}_{\geq 0}$ and $\alpha \in \mathbb{R}_{\geq 0}$ determine the maximum strength and the duration, respectively, of the effect of $l_{2}(\cdot)$ in the closed loop system.

Clearly, other choices are possible. For instance it may be of interest to evaluate the performance index $g(\cdot)$ only within a finite number of time windows. It this case, a suitable choice is $p(t)=B \operatorname{win}_{\mathcal{W}}(t)$ with

$$
\operatorname{win}_{\mathcal{W}}(t)= \begin{cases}1 & \text { if } t \in\left[t_{i}, t_{i}+L_{i}\right],\left(t_{i}, L_{i}\right) \in \mathcal{W} \\ 0 & \text { otherwise }\end{cases}
$$

where a generic element $w=(\bar{t}, \bar{L}) \in \mathcal{W} \subset \mathbb{R}_{\geq t_{0}} \times \mathbb{R}_{\geq 0}$ identifies a time window of length $\bar{L}$ starting at time $\bar{t}$, and the set of time windows has a finite cardinality, i.e., $|\mathcal{W}|<+\infty$ where, for a generic set $\mathcal{A},|\mathcal{A}|$ denotes its cardinality. Consequently, the bound $\mathcal{L}(t) \leq \sum_{i=1}^{|\mathcal{W}|} B L_{i}$ holds and, thus, Assumption 1 is satisfied.

\section{B. Convergence and Lyapunov Stability}

It is worth noting that, during the transient phase, the proposed MPC controller can potentially drive the state away from the origin in order to minimize the secondary stage cost $l_{2}(\cdot)$. This fact, which is the desired behavior, implies that stability, in the classic Lyapunov sense (see, e.g., Definition 4.1 of [13]), cannot in general be established even when convergence to the origin is guaranteed.

More precisely, in a classic MPC setting, under some extra assumptions (see e.g. [8]), it is possible to show that the typical function $V(\cdot)$ (i.e., (8) with $l_{2}(\cdot)=0$ and

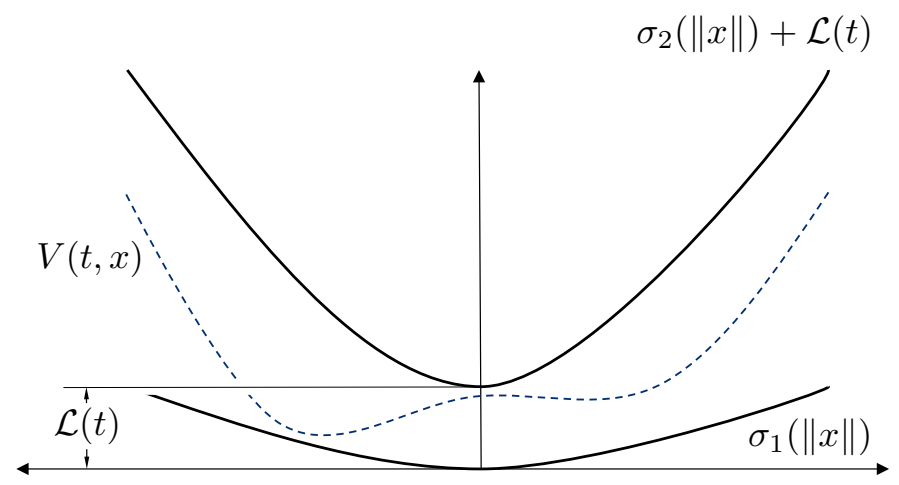

Fig. 1. Illustrative figure of the effect of $\mathcal{L}(t)$ on the upper and lowed bounds of the value function.

$\mathcal{L}(\cdot)=0$ ), can be uniformly lower and upper bounded by class- $\mathcal{K}$ functions ${ }^{3} \sigma_{1}(\|x\|)$ and $\sigma_{2}(\|x\|)$, respectively. This observation, together with the fact that $V(\cdot)$ decreases along the closed loop trajectory, is used to prove asymptotic stability of the origin (see, e.g., Theorem 4.16 of [13]). In the proposed approach such uniform class- $\mathcal{K}$ upper bound cannot be established. In fact, even if $\sigma_{1}(x)$ and $\sigma_{2}(x)$ are available for the original problem (i.e., $l_{2}(\cdot)=0$ and $\mathcal{L}(\cdot)=0$ ), due to the extra term $\mathcal{L}(\cdot)$ in (8), the upper bound is shifted up by the value of $\mathcal{L}(t)$, as graphically shown in Fig. 1 losing the properties of the class- $\mathcal{K}$ functions. Thus, in general, only convergence, and not stability, can be established.

Notice however, that guaranteeing only convergence, and not Lyapunov stability, is not a new in the MPC literature, see e.g., [5], [6], [4]. This is justified by the fact that in many practical applications, especially for constrained system, Lyapunov stability is impossible to satisfy, where it is possible to design a control law that drives the system to the origin. We refer to [1] for a nice insight on the topic.

\section{SIMULATION RESULTS}

Consider the following model

$$
\dot{x}(t)=u(t), \quad x(0)=x_{0}, \quad t \geq t_{0}
$$

where $x(t) \in \mathbb{R}^{2}$ denotes the position of a simple holonomic vehicle and the control input $u(t) \in \mathbb{R}^{2}$, its linear velocity, is constrained as $\|u(t)\|_{\infty} \leq 3$. Given that the system is linear with polytopic constraints, and choosing the following quadratic stage cost

$$
l(x, u)=\|x\|^{2}+\|u\|^{2},
$$

a stabilizing MPC controller can be designed as follows:

(i) compute a linear controller $u=K x$ that stabilizes the origin of the unconstrained system (11), e.g., using the Linear Quadratic Regulator (LQR) approach,

\footnotetext{
${ }^{3} \mathrm{~A}$ continuous function $\alpha:[0, a) \rightarrow[0, \infty)$ is said to belong to class $\mathcal{K}$, or to be a class- $\mathcal{K}$ function, if it is strictly increasing and $\alpha(0)=0$. It is said to belong to class $\mathcal{K}_{\infty}$, or to be a class- $\mathcal{K}_{\infty}$ function, if it belongs to class $\mathcal{K}, a=\infty$, and $\alpha(s) \rightarrow 0$ as $s \rightarrow 0$
} 


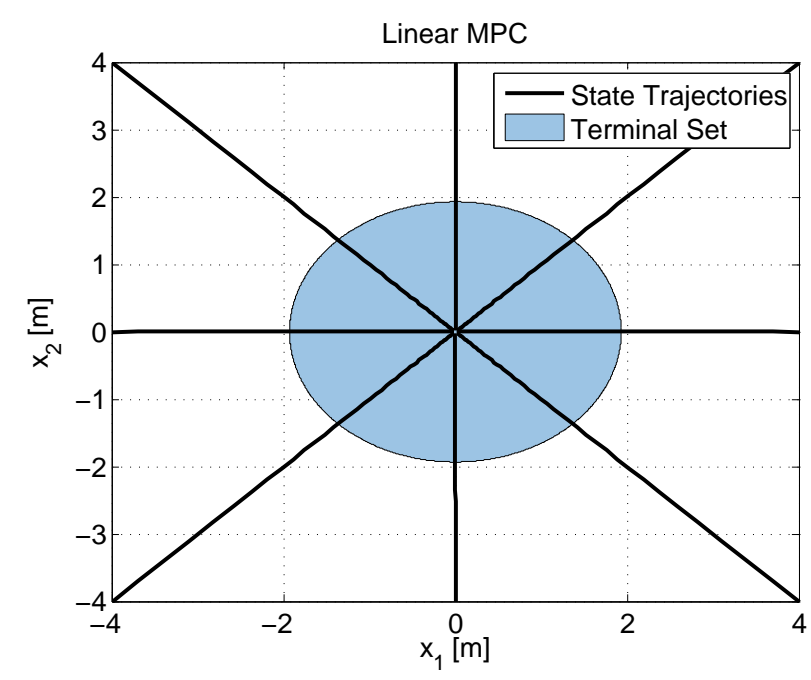

Fig. 2. System (11) in closed loop with the computed linear MPC controller, where the observability index is not considered.

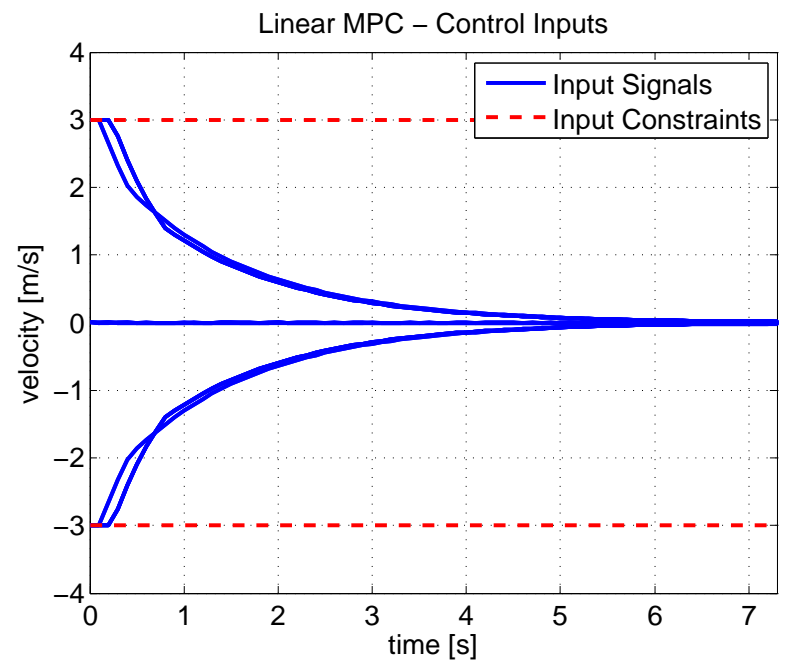

Fig. 3. Input trajectories of system (11) in closed loop with the computed linear MPC controller, where the observability index is not considered.

(ii) find the matrix $P$ that uniquely solve the Algebraic Riccati Equation (ARE)

$$
(A+B K)^{\prime} P+P(A+B K)+\left(I+K^{\prime} K\right)=0,
$$

(iii) define the terminal cost $F(x)=x^{\prime} P x$,

(iv) define the terminal set $\mathcal{X}_{f}$ to be the largest level set of $F(x)$ contained inside the constraints.

This procedure results in $F(x)=x^{\prime} P x$, $\mathcal{X}_{f}=\left\{x: x^{\prime} P x \leq 9\right\}$ with $P=\left(\begin{array}{cc}2.4142 & 0 \\ 0 & 2.4142\end{array}\right)$.

Choosing the horizon length $T=1$ second and $\mathcal{T}=\left\{n 0.1, n \in \mathbb{N}_{\geq 0}\right\}$, Fig. 2 and Fig. 3 show the state and input trajectories, respectively, of system (11) in closed loop with the resulting MPC controller associated to the initial conditions $x_{0} \in\left\{[ \pm 4, \pm 4]^{\prime},[0, \pm 4]^{\prime},[ \pm 4,0]^{\prime}\right\}$, $t_{0}=0$. For simulation purposes, system (11) was discretized

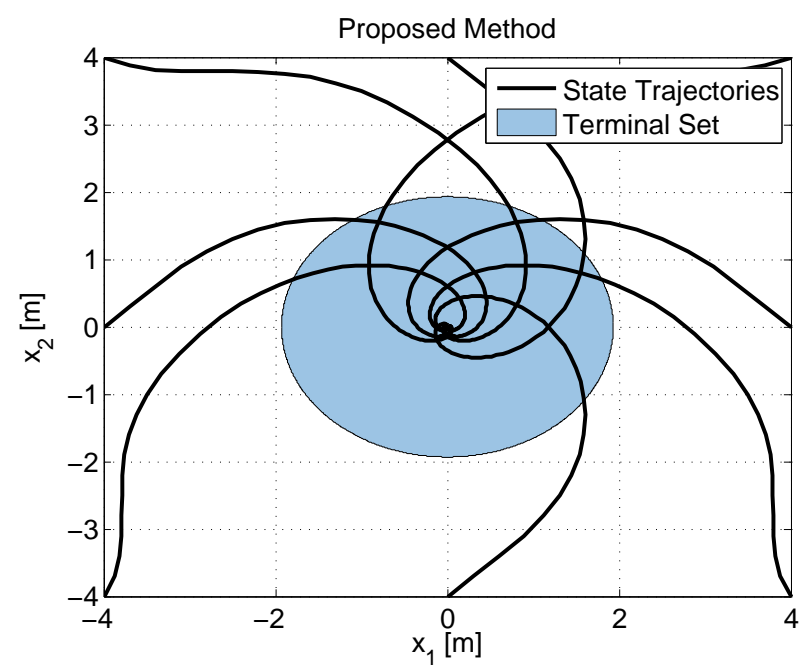

Fig. 4. System (11) in closed loop with the proposed MPC law, and with an observability index considered in the stage cost function.

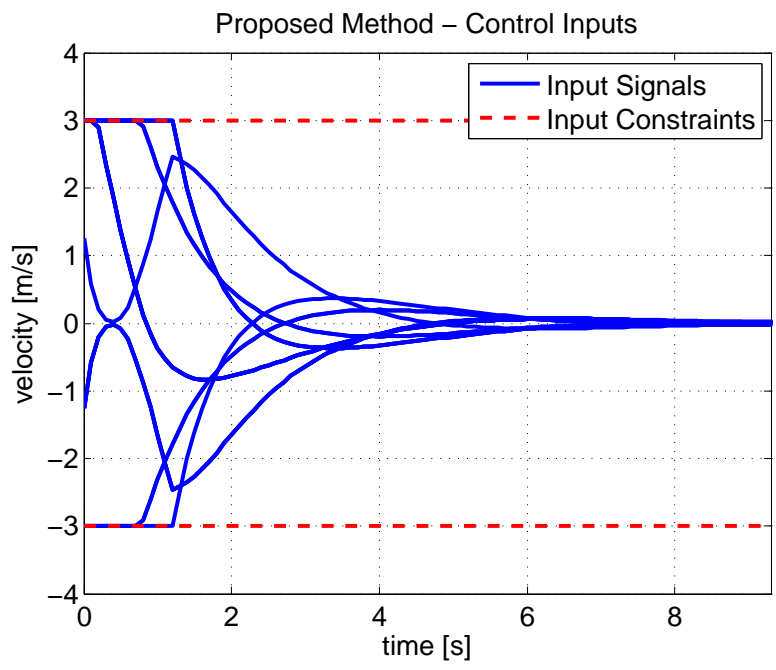

Fig. 5. Input trajectories of system (11) in closed loop with the proposed MPC law, and with an observability index considered in the stage cost function.

with a sampling time of 0.1 seconds.

Now, consider that the position of vehicle is continuously observed by an omnidirectional camera centered at the origin. Then, the observation model can be defined as

$$
y(t)=\frac{x(t)}{\|x(t)\|},
$$

where $y(t) \in \mathbb{R}^{2}$ is a bearing only observation, which provides information about the direction of the vehicle but not about the distance.

In order to obtain an index of observability for the system under consideration we consider, similarly to [2], the observability matrix

$$
\mathcal{O}(x, u)=\frac{\partial}{\partial x}\left(\begin{array}{l}
y \\
\dot{y}
\end{array}\right)
$$




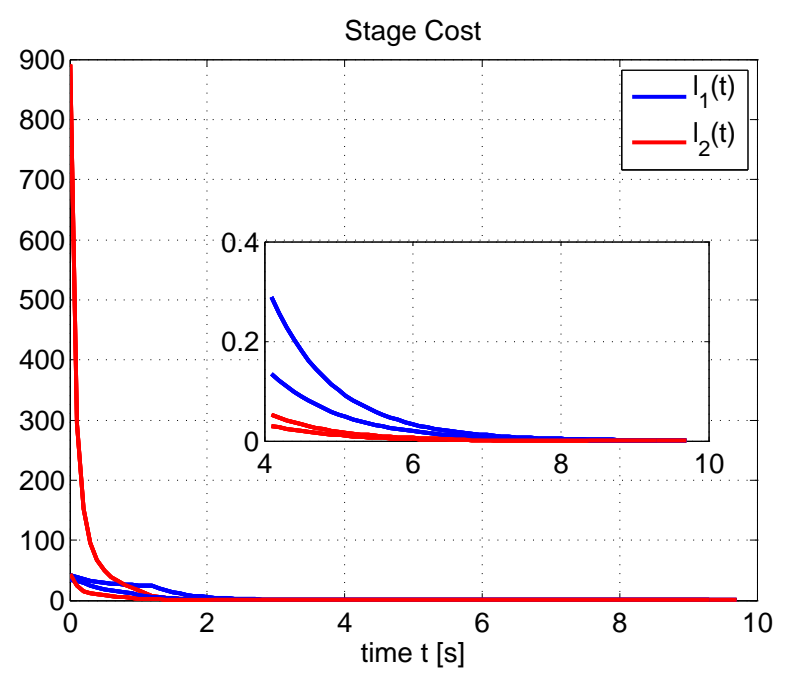

Fig. 6. The primarily and secondarily stage cost associated with the trajectories of system (11) in closed loop with the proposed MPC law.

and we use the fact that the system is locally observable at a given state and input pair $(\bar{x}, \bar{u})$ if $\mathcal{O}(\bar{x}, \bar{u})$ is full rank. Let $\sigma_{\min }(A)$ and $\sigma_{\max }(A)$ denote the minimum and maximum singular value of a generic matrix $A$. To obtain a measure of the degree of observability, one possibility is to use the index $1 / \sigma_{\min }(\mathcal{O}(x, u))$, which increases as $\mathcal{O}$ get close to singularity and becomes infinity when $\mathcal{O}$ loses rank. Another index of interest is the condition number of $\mathcal{O}$, i.e., $\kappa(\mathcal{O}):=\sigma_{\max }(\mathcal{O}) / \sigma_{\min }(\mathcal{O})$, which broadly speaking, provides a measure of the difference of the quality of observability of the state components, where $\kappa(\mathcal{O})=1$ if the all the state components have the same quality of observability.

Analyzing the observability matrix of the system (11)-(13) considered in this example, it is possible to see that the state is locally not observable when the velocity vector is parallel to the position vector, i.e., when the vehicle moves towards or way from the camera, which means that all the closed loop trajectories sketched in Fig. 2 are not observable. This condition, well known in computer vision, comes from the fact that we cannot observe the distance between the camera and the vehicle and, thus, only the parallax of observation (i.e., observation along different lines of sight) provides meaningful information on the state.

In view of the above remarks, we use the method proposed in this paper to drive the vehicle to the origin maintaining with some degree of observability. To this end, we redefine the stage cost as (5) as (12) where $l_{1}(x, u)=\|x\|^{2}+\|u\|^{2}$ and $l_{2}(t, x, u)=$ $\operatorname{sat}_{10100}\left(1 / \sigma_{\text {min }}^{2}(\mathcal{O}(x, u))+(\kappa(\mathcal{O}(x, u))-1)^{2}\right) e^{-t}$.

Fig. 4 and Fig. 5 show the associated state and input closed loop trajectories, respectively, and in Fig. 6 the evolution of the primarily and secondarily stage cost is displayed. As result, the vehicle is driven to the origin trough curvilinear trajectories, which bring more information for the estimation of the position of the vehicle.

\section{CONCLUSION AND FUTURE WORK}

This paper presents a state feedback sampled data MPC scheme for nonlinear time varying continuous time system. An additional stage cost, which in general is not a measure of the distance to the set point, is used to influence the transient behavior of the controlled system. Convergence to the origin is guaranteed with the only extra assumption of integrability of the additive stage cost in the interval $[t,+\infty)$, which as shown in Section IV-A, can in general be enforced. This MPC scheme is applied to a control problem, taken from the literature, where an MPC controller is designed to drive the state to the origin while penalizing weakly observable trajectories.

\section{REFERENCES}

[1] F. A. C. C. Fontes. Discontinuous feedbacks, discontinuous optimal controls, and continuous-time model predictive control. International Journal of Robust and Nonlinear Control, 13(3-4):191-209, Mar. 2003.

[2] C. Böhm, R. Findeisen, and F. Allgoewer. Avoidance of Poorly Observable Trajectories: A predictive control perspective. In Proceedings of the 17th IFAC World Congress, pages 1952-1957, 2008.

[3] H. Chen and F. Allgöwer. A Quasi-Infinite Horizon Nonlinear Model Predictive Control Scheme with Guaranteed Stability. Automatica, 34(10):1205-1217, Oct. 1998.

[4] R. Findeisen, L. Imsland, F. Allgower, and B. A. Foss. State and output feedback nonlinear model predictive control: An overview. European journal of control, 9(2-3):190-206, 2003.

[5] F. Fontes and L. Magni. Min-max model predictive control of nonlinear systems using discontinuous feedbacks. IEEE Transactions on Automatic Control, 48(10):1750-1755, Oct. 2003.

[6] F. Fontes, L. Magni, and E. Gyurkovics. Sampled-Data Model Predictive Control for Nonlinear Time-Varying Systems: Stability and Robustness. volume 358 of Lecture Notes in Control and Information Sciences, pages 115-129. Springer Berlin / Heidelberg, 2007.

[7] F. A. Fontes. A general framework to design stabilizing nonlinear model predictive controllers. Systems \& Control Letters, 42(2):127143, Feb. 2001.

[8] G. Grimm, M. Messina, S. Tuna, and A. Teel. Model predictive control: for want of a local control Lyapunov function, all is not lost. IEEE Transactions on Automatic Control, 50(5):546-558, May 2005.

[9] L. Grüne. Analysis and Design of Unconstrained Nonlinear MPC Schemes for Finite and Infinite Dimensional Systems. SIAM Journal on Control and Optimization, 48(2):1206, Mar. 2009.

[10] L. Grune and J. Pannek. Nonlinear model predictive control: theory and algorithms. 2011.

[11] L. Grüne, J. Pannek, M. Seehafer, and K. Worthmann. Analysis of Unconstrained Nonlinear MPC Schemes with Time Varying Control Horizon. SIAM Journal on Control and Optimization, 48(8):4938, Oct. 2010.

[12] A. Jadbabaie, J. Yu, and J. Hauser. Unconstrained receding-horizon control of nonlinear systems. IEEE Transactions on Automatic Control, 46(5):776-783, May 2001.

[13] H. K.Khalil. Nonlinear Systems. Prentice Hall,Inc., third edition, 2002.

[14] D. Mayne, J. Rawlings, C. Rao, and P. Scokaert. Constrained model predictive control: Stability and optimality. Automatica, 36(6):789814, June 2000.

[15] M. Morari and J. H. Lee. Model predictive control: Past, present and future. Computers \& Chemical Engineering, 23(4):667-682, 1999.

[16] J. A. Primbs and V. Nevistic. A New Approach to Stability Analysis for Constrained Finite Receding Horizon Control without End Constraints, 1997.

[17] J. B. Rawlings and D. Q. Mayne. Model Predictive Control Theory and Design. Nob Hill Pub., 2009.

[18] M. Reble, D. E. Quevedo, and F. Allgower. A unifying framework for stability in MPC using a generalized integral terminal cost. In American Control Conference (ACC), 2012, pages 1211-1216, June 2012. 